URszula LeDZEwicz (Edwardsville, IL)

Vignon Oussa (Edwardsville, IL)

Heinz SchäTtLeR (St. Louis, MO)

\title{
OPTIMAL SOLUTIONS FOR A MODEL OF TUMOR ANTI-ANGIOGENESIS WITH A PENALTY ON THE COST OF TREATMENT
}

Abstract. The scheduling of angiogenic inhibitors to control a vascularized tumor is analyzed as an optimal control problem for a mathematical model that was developed and biologically validated by Hahnfeldt et al. [Cancer Res. 59 (1999)]. Two formulations of the problem are considered. In the first one the primary tumor volume is minimized for a given amount of angiogenic inhibitors to be administered, while a balance between tumor reduction and the total amount of angiogenic inhibitors given is minimized in the second formulation. The optimal solutions to both problems are presented and compared.

1. Introduction. Tumor anti-angiogenesis is a cancer treatment approach that targets the vasculature of a growing tumor. A solid tumor, after going through a state of avascular growth, at the size of about $2 \mathrm{~mm}$ in diameter, starts the process of angiogenesis [12] to recruit surrounding, mature, host blood vessels needed for its supply of nutrients. The lining of these newly developing blood vessels consists of endothelial cells and the tumor produces vascular endothelial growth factor (VEGF) to stimulate their growth [20] as well as inhibitors to suppress it [13]. Anti-angiogenic treatments rely on these mechanisms by bringing in external angiogenic inhibitors (e.g., endostatin) that target endothelial cells and block their growth. This indirectly effects the tumor which, ideally, deprived of necessary nutrition, regresses. Since, contrary to traditional chemotherapy, this treatment does

2000 Mathematics Subject Classification: 49J15, 93C50.

Key words and phrases: optimal control, bang-bang and singular controls, cancer treatments, angiogenic inhibitors. 
not target the quickly duplicating and genetically unstable cancer cells, but attacks genetically stable normal cells instead, it was observed that no resistance to the anti-angiogenic agents developed in experimental cancer [4]. For this reason tumor anti-angiogenesis has been called a therapy "resistant to resistance" that provides a new hope for the treatment of tumor type cancers [18].

Naturally, tumor anti-angiogenesis became an active area of research in the last ten years not only in medicine $[10,17,16,19]$, but also in other disciplines including mathematical biology, $[1,9,11,14,30]$, and several models describing the dynamics of angiogenesis have been formulated. Some of these attempt to fully reflect the complexity of the biological processes, e.g., $[2,3]$, and allow for large scale simulations, but are not amenable to mathematical analysis since theoretical techniques from such fields as dynamical systems or optimal control theory can only effectively be used on low dimensional systems. Hahnfeldt, Panigrahy, Folkman and Hlatky [17], a group of researchers then at Harvard Medical School, in 1999 developed and biologically validated such a two-dimensional model of ordinary differential equations for the interactions between the tumor volume, $p$, and the carrying capacity of the vasculature, $q$. The latter is defined as the maximum tumor volume sustainable by the vascular network. Since it is largely determined by the volume of endothelial cells, we also refer to this as the endothelial support of the tumor for short. This model, and the underlying spatial analysis carried out in that research, has seen various modifications, for example, by d'Onofrio and Gandolfi [9] at the European Institute of Oncology in Milan, or by Ergun, Camphausen and Wein [11] at the Cancer Research Institute at NIH, and still is an area of active research [1, 8, 14, 23, 25, 32, 34].

In this paper, we consider two related formulations of tumor anti-angiogenesis as optimal control problems for the original model by Hahnfeldt et al. [17]. In the first formulation, mathematically already analyzed in [26], the primary tumor volume is minimized with a given amount of anti-angiogenic agents to be administered as constraint. It has been shown in [26] that optimal controls are concatenations of at most five pieces of the form 0asa0 where 0 denotes an interval when no inhibitors are administered, $a$ denotes an interval when inhibitors are given at an a priori determined maximum dose $a$, and $s$ represents an interval where the optimal control is singular and follows an explicitly computed time varying feedback control that takes values strictly between 0 and the maximum value $a$. (Depending on the specific initial condition, not all of these pieces need to be present.) Based on this characterization, the numerical computation of optimal controls and trajectories is easily accomplished and a complete synthesis of optimal controls and trajectories for all initial conditions was given in [26]. 
In this formulation, since the total amount of angiogenic inhibitors is taken as a constraint, it is intuitively clear, and easily verified analytically, that optimal controls will use up all available agents. Consequently, even if there is only a marginal reduction in tumor size to be gained, if inhibitors are still available, they will be used regardless of cost or potential side effects. Given the fact that inhibitors are very expensive biological agents, this need not be a cost effective strategy. Therefore, in this paper we modify the above problem formulation to better balance the total amount of inhibitors given with the benefit to be gained in tumor reduction. Rather than specifying the total amount of inhibitors a priori, we incorporate this amount as a penalty term in the objective and then minimize a weighted average between the inhibitors given and the minimum tumor volume. Clearly, other kind of objectives can be considered as well, and they all may lead to both qualitatively and quantitatively different results. Our formulation naturally connects with the problem already solved in [26] and preserves the qualitative structure of solutions in the form Oasa0, but solutions differ in their quantitative aspects. Also, a bifurcation analysis of the structure of optimal trajectories depending on the weight given to the angiogenic inhibitors in the objective is easily accomplished and will be illustrated with numerical results. Our results here complement those in $[23,24]$ obtained for a simplified version of the underlying model considered in this paper.

2. Anti-angiogenic therapy: maximizing tumor reduction. We briefly review the underlying mathematical model that was developed and biologically validated by Hahnfeldt, Panigrahy, Folkman and Hlatky in [17]. State variables are the primary tumor volume, $p$, and the carrying capacity of the vasculature, $q$. Tumor growth is modelled by a Gompertzian growth function with carrying capacity $q$, i.e.,

$$
\dot{p}=-\xi p \ln (p / q)
$$

where $\xi$ denotes a tumor growth parameter. The dynamics for the endothelial support is of the form

$$
\dot{q}=b p-\left(\mu+d p^{2 / 3}\right) q-G u q
$$

where $b p$ models the stimulation of endothelial cells by the tumor and the term $d p^{2 / 3} q$ models endogenous inhibition of the tumor. The exponent $2 / 3$ arises since these inhibitors are released through the tumor surface and thus the tumor's volume needs to be scaled to its surface area (the primary tumor is modelled as a sphere in [17]). The product $p^{2 / 3} q$ then describes the interactions of inhibitors with the volume of endothelial cells. The coefficients $b$ and $d$ are growth constants. The terms $\mu q$ and Guq describe, respectively, loss to the carrying capacity through natural causes (death of endothelial 
cells etc.), and loss due to extra outside inhibition. The variable $u$ represents the control in the system and corresponds to the angiogenic dose rate while $G$ is a constant that represents the anti-angiogenic killing parameter. Generally $\mu$ is small and often this term is negligible compared to the other factors and thus in the literature sometimes $\mu$ is set to 0 in this equation. The following result from [9] guarantees the existence and positivity of solutions for all times and arbitrary controls $u$.

Proposition 1 ([9]). For any non-negative, locally bounded, Lebesgue measurable function $u$ and arbitrary positive initial conditions $p_{0}$ and $q_{0}$ the solution $(p, q)$ to equations (1) and (2) with initial conditions $p_{0}$ and $q_{0}$ exists for all times $t \geq 0$ and both $p$ and $q$ remain positive.

It is shown in [9] that the uncontrolled model $(u=0)$ has a unique, globally asymptotically stable equilibrium point given by $\bar{p}=\bar{q}=((b-\mu) / d)^{3 / 2}$ which for realistic values of the parameters naturally is not biologically viable. Adding a control term $u=a$, for large enough $a, G a>b-\mu$, this globally asymptotically stable node ceases to exist and all trajectories for the corresponding system converge to the origin in infinite time. This, in principle, would be the desired situation since, at least theoretically, it allows for eradication of the tumor using a constant dose $u=a$ for all time. But clearly this is not a feasible strategy because of limits on the total amount of inhibitors and potential side effects. The problem of how to administer a given amount of inhibitors to achieve the "best possible" effect thus arises naturally.

One possible formulation, considered first in [11] and then taken up by us in $[25,26,27]$, is to solve the following optimal control problem: for a free terminal time $T$, minimize the value $p(T)$ subject to the dynamics (1) and (2) over all Lebesgue measurable functions $u:[0, T] \rightarrow[0, a]$ that satisfy a constraint on the total amount of anti-angiogenic inhibitors to be administered,

$$
\int_{0}^{T} u(t) d t \leq A .
$$

The upper limit $a$ in the definition of the control set $U=[0, a]$ is a previously determined maximum dose at which inhibitors can be given. Note that the time $T$ in this formulation does not correspond to a therapy period, but instead is the time when the maximum tumor reduction achievable with the given overall amount $A$ of inhibitors is being realized. Alternatively, the same scheduling problem could be considered over an a priori prescribed therapy horizon $T_{t h}$ so that the final time $T_{t h}$ is fixed. But this leads to a slightly different optimal control problem, pursued by A. Swierniak et al. (e.g., $[32,34])$. Mathematically, it is more convenient to adjoin the constraint 
(3) as a third variable and define the problem in $\mathbb{R}^{3}$. Hence we consider the following optimal control problem:

(P1) For a free terminal time $T$, minimize the value

$$
J_{1}(u)=p(T)
$$

subject to the dynamics

$$
\begin{array}{ll}
\dot{p}=-\xi p \ln (p / q), & p(0)=p_{0}, \\
\dot{q}=b p-\left(\mu+d p^{2 / 3}\right) q-G u q, & q(0)=q_{0}, \\
\dot{y}=u, & y(0)=0,
\end{array}
$$

over all piecewise continuous functions $u:[0, T] \rightarrow[0, a]$ for which the corresponding trajectory satisfies $y(T) \leq A$.

For the simulations shown in this paper we use the following parameter values that are taken from [17]: The variables $p$ and $q$ are volumes measured in $\mathrm{mm}^{3} ; \xi=\frac{0.192}{\ln 10}=0.084$ per day (adjusted to the natural logarithm), $b=5.85$ per day, $d=0.00873$ per $\mathrm{mm}^{2}$ per day, $G=0.15 \mathrm{~kg}$ per $\mathrm{mg}$ of dose per day, and for illustrative purposes we chose a small positive value for $\mu$, $\mu=0.02$ per day. Finally, we have chosen $a=75$ and $A=300$ for the limits on the control. These values are merely used for numerical illustrations; the mathematical results presented here are valid in general under the reasonable assumption that $G a>b-\mu>0$, i.e., that the parameters related to outside inhibition are able to overcome the net effect of "birth" minus "death" of the endothelial support.

The optimal controls for the problem considered in Section 3 are based on the solutions of this problem and therefore, based on earlier results [17], we first summarize the general structure of optimal trajectories and then proceed to a precise description of the optimal controls. However, in order to exclude discussions about the structure of optimal controls in regions where the model does not represent the underlying biological problem, we restrict our discussions to the biologically realistic domain

$$
\mathcal{D}=\{(p, q): 0<p \leq \bar{p}, 0<q \leq \bar{q}\} .
$$

Theorem 1 ([26]). For any initial condition $\left(p_{0}, q_{0}\right) \in \mathcal{D}$, optimal controls are at most concatenations of the form 0asa0 where 0 denotes an interval along which the optimal control is given by the constant control $u=0$, that is, no inhibitors are given, a represents an interval along which the optimal control is given by the constant control $u=a$ at full dose, and $s$ denotes an interval along which the optimal control follows a time-varying feedback control (to be specified below), the so-called singular control. This control is only optimal while the system follows a particular curve $\mathcal{S}$ in the $(p, q)$-space, the optimal singular arc. However, depending on the initial condition $\left(p_{0}, q_{0}\right)$, not all of these intervals need to be present in a specific 
solution. For the biologically most relevant initial conditions typically optimal controls have the form bso where $b$ denotes an interval along which the optimal control is given by either a or 0 depending on the initial condition.

Despite their name, which is related to some classical control literature from the sixties (e.g., [7]), singular controls are to be expected in a synthesis of optimal controls for a problem of the type (P1) for nonlinear models [5]. The singular control and the geometry of the corresponding trajectory $\mathcal{S}$ are the most important part of the design of optimal protocols and the formulas given below (whose derivations can be found in $[25,26]$ ) are essential in the construction of a synthesis of optimal controls and trajectories.

Proposition $2([26])$. The singular curve $\mathcal{S}$ lies in the sector $\{(p, q)$ : $\left.x_{1}^{*} q<p<x_{2}^{*} q\right\}$ where $x_{1}^{*}$ and $x_{2}^{*}$ are the unique zeroes of the equation

$$
\varphi(x)=\frac{b}{d} x(\ln x-1)+\frac{\mu}{d}=0
$$

and satisfy $0 \leq x_{1}^{*}<1<x_{2}^{*} \leq e$. In the variables $(p, x)$ with $x=p / q$, the singular curve $\mathcal{S}$ can be parameterized in the form

$$
p^{2}+\varphi(x)^{3}=0 \quad \text { for } x_{1}^{*}<x<x_{2}^{*} .
$$

The singular control keeps the system on the singular curve and is given as a feedback function of $x$ in the form

$$
u_{\sin }(x)=\frac{1}{G}\left[\left(\frac{1}{3} \xi+b x\right) \ln x+\frac{2}{3} \xi\left(1-\frac{\mu}{b x}\right)\right] .
$$

There exists exactly one connected arc on the singular curve $\mathcal{S}$ along which the singular control is admissible, i.e., satisfies the bounds $0 \leq u_{\sin }(x) \leq a$. This arc is defined over an interval $\left[x_{l}^{*}, x_{u}^{*}\right]$ where $x_{l}^{*}$ and $x_{u}^{*}$ are the unique solutions to the equations $u_{\sin }\left(x_{l}^{*}\right)=0$ and $u_{\sin }\left(x_{u}^{*}\right)=a$ and these values satisfy $x_{1}^{*}<x_{l}^{*}<1<x_{u}^{*}<x_{2}^{*}$.

The two graphs given in Fig. 1 illustrate the proposition for the parameter values from [17] specified earlier. Fig. 1(a) shows the plot for the singular control defined by (11) also indicating the values $x_{l}^{*}$ and $x_{u}^{*}$ where the control saturates at $u_{\sin }(x)=0$ and $u_{\sin }(x)=a$. Fig. $1(\mathrm{~b})$ shows the singular curve $\mathcal{S}$ given by formula (10). Saturation of the singular control at $x_{l}^{*}$ and $x_{u}^{*}$ restricts the admissible part of this petal-like curve to the portion lying between the lines $p=x_{l}^{*} q$ and $p=x_{u}^{*} q$. This portion is marked with a solid line in Fig. 1(b). The qualitative structures shown in Fig. 1 are generally valid for arbitrary parameter values, both for the control and the singular curve. But naturally, with decreasing values for the upper control limit $a$ the admissible portion shrinks.

The admissible singular arc becomes the centerpiece of the synthesis of optimal solutions given in Fig. 2. The important curves for the synthesis 

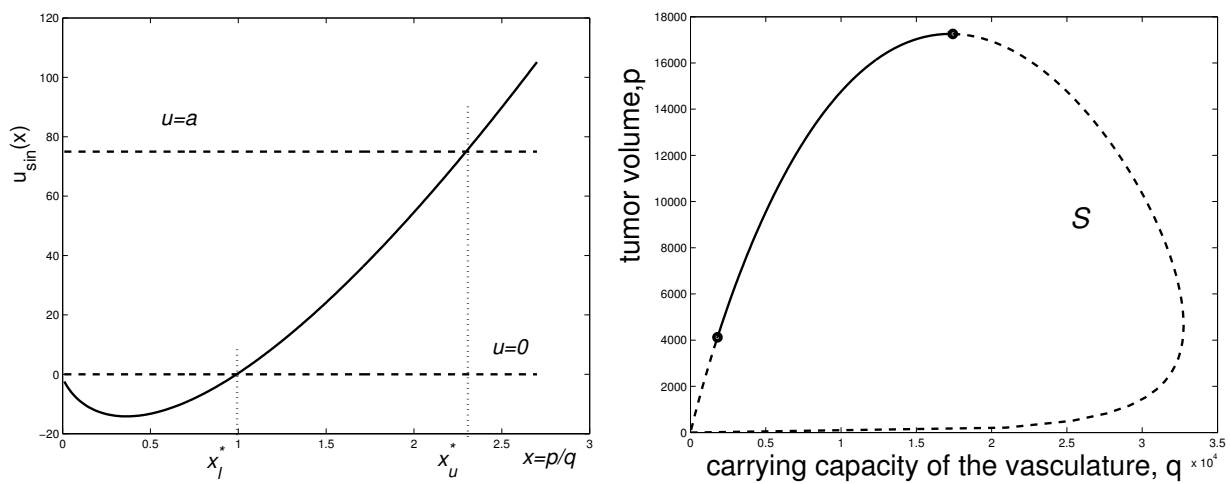

Fig. 1. (a) Singular control and (b) admissible singular arc

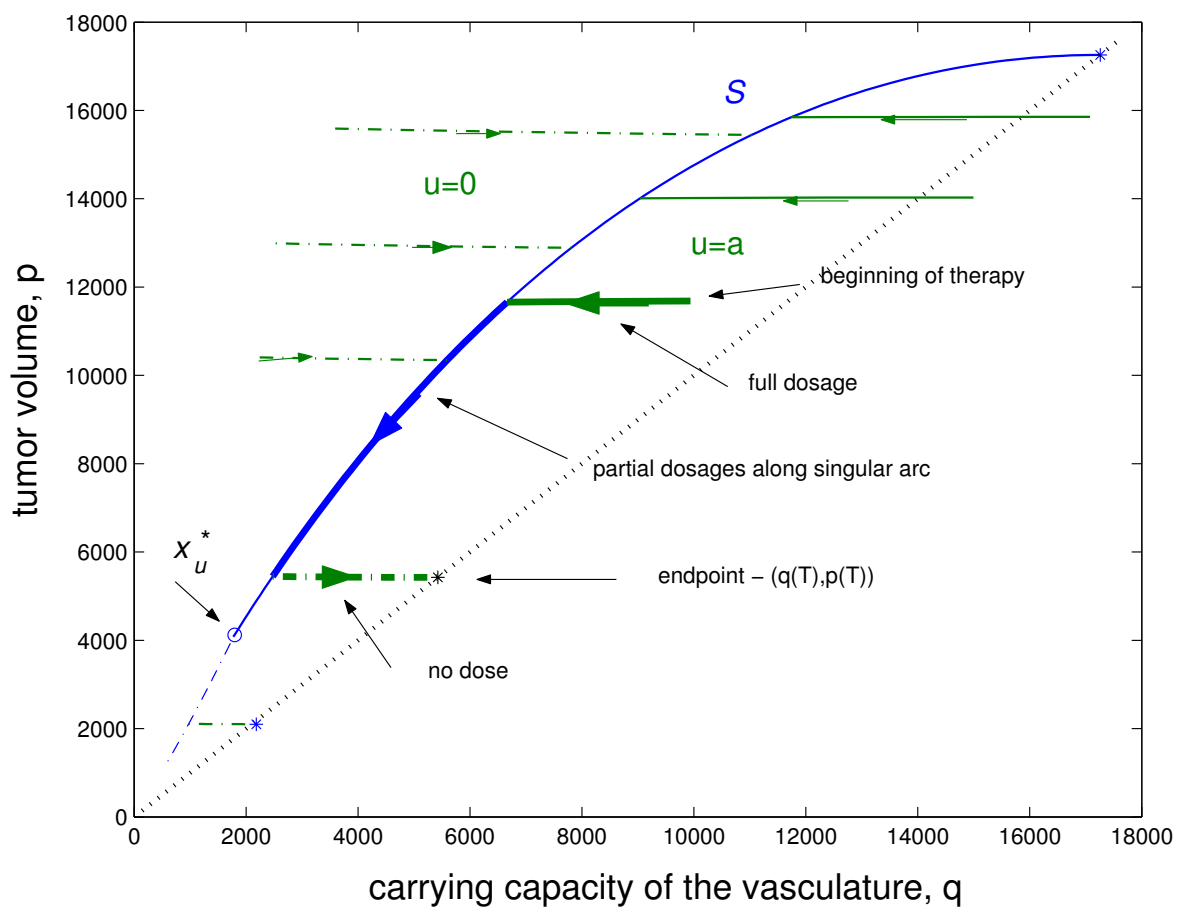

Fig. 2. Synthesis of optimal controlled trajectories

are the admissible portions of the singular curve (solid blue curve), portions of trajectories corresponding to the constant controls $u=0$ (dashed-dot green curves) and $u=a$ (solid green curves), and the line $p=q$ (dotted black line) where the trajectories achieve the maximum tumor reduction. These diagrams represent the optimal trajectories as a whole and each of the different curves gives a different optimal trajectory depending on the 
actual initial condition. The thick curves in the graphs mark one specific such trajectory. For this case the initial value $p_{0}$ for the tumor volume and $q_{0}$ for the endothelial support are high and require to immediately start the treatment. The optimal trajectory therefore initially follows the curve corresponding to the control $u=a$. Note that, although inhibitors are given at full dose along this curve, this shows very little effect on the number of the cancer cells in a sense of decrease. The reason is that during this interval the inhibitors drive down the vascular support and in this way prevent further growth of the tumor that otherwise, enabled by its ample vascular support, would occur. Once the trajectory corresponding to the full dose hits the singular arc $S$, according to our analysis it is then no longer optimal to give full dose and the optimal controls here switch to the singular control and the optimal trajectory follows the singular arc. Only at this point does a significant tumor reduction commence. Ignoring some special cases that are due to saturation of the singular control along this arc and are described in [26], the optimal control will now follow the singular arc until all inhibitors are exhausted according to the condition that $y(T)=A$. When the inhibitors have been exhausted, therapy is over. But, due to after-effects, the tumor is still shrinking as long as $p(t)>q(t)$. Hence the maximum tumor reduction is only realized along a trajectory for the control $u=0$ as this trajectory crosses the diagonal $p=q$. The corresponding time $T$ is then the limit of the horizon considered in the problem formulation (P1).
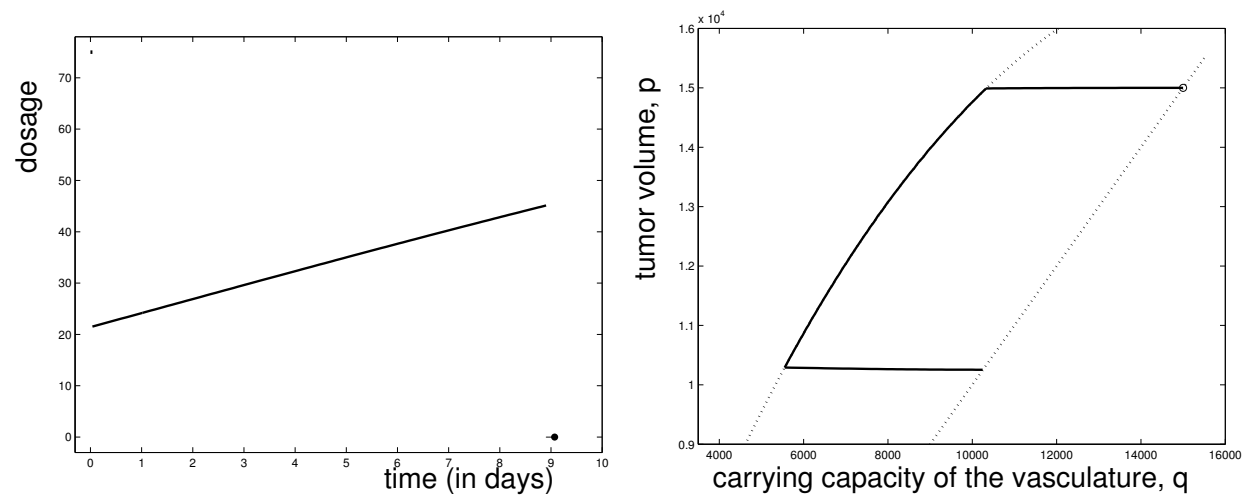

Fig. 3. (P1)-optimal control (left) and corresponding optimal trajectory (right)

Fig. 3 gives the optimal control and its corresponding trajectory for the initial conditions $\left(p_{0}, q_{0}\right)=\left(15,000 \mathrm{~mm}^{3} ; 15,000 \mathrm{~mm}^{3}\right)$. In this case the control starts with full dose $u=a=75$ for $t_{1}=0.04$ days and then follows the singular arc until $t_{2}=8.91$ days when all inhibitors become exhausted. The maximum tumor reduction is realized at time $T=9.09$ days with the value $p_{\min }=10,254 \mathrm{~mm}^{3}$. 


\section{Anti-angiogenic therapy: minimizing tumor volume with a} cost on treatment. The objective chosen in problem (P1) a priori puts a cost (that can be viewed in terms of a price, but also as limiting potential side effects) on the usage of angiogenic inhibitors in terms of the isoperimetric constraint (3). Naturally, in this case optimal solutions exhaust all available inhibitors regardless of incremental benefits. We now consider a modification of this model where we balance the amount of inhibitors used with a reduction in tumor size over time.

(P2) For a free terminal time $T$, minimize

$$
J_{2}(u)=p(T)+\kappa \int_{0}^{T} u(t) d t
$$

over all Lebesgue measurable functions $u:[0, T] \rightarrow[0, a]$ subject to

$$
\begin{array}{ll}
\dot{p}=-\xi p \ln (p / q), & p(0)=p_{0}, \\
\dot{q}=b p-\left(\mu+d p^{2 / 3}\right) q-G u q, & q(0)=q_{0} .
\end{array}
$$

In the objective, as in model $(\mathrm{P} 1)$, the term $\int_{0}^{T} u(t) d t$ represents the total amount of anti-angiogenic treatment administered. But here this term is viewed as a measure for the cost of the treatment or related to side effects. In this formulation, we thus attempt to balance the effectiveness of the treatment with cost and side-effects through the positive weight $\kappa$ at this integral.

We show that the qualitative structure of solutions does not change, i.e., optimal controls for problem (P2) still follow the regimen 0asa0 and thus optimal controls again can very effectively and fast be computed numerically. The differences to problem (P1) lie in the conditions for termination of anti-angiogenic treatment. First-order necessary conditions for optimality of a control $u$ are given by the Pontryagin Maximum Principle [29, 7]. It is easily seen that the problem is normal and thus these conditions can be formulated as follows: if $u_{*}$ is an optimal control defined over the interval $[0, T]$ with corresponding trajectory $\left(p_{*}, q_{*}\right)$, then there exists an absolutely continuous co-vector, $\lambda:[0, T] \rightarrow\left(\mathbb{R}^{2}\right)^{*}$ (which we write as row-vector) that satisfies the adjoint equations with transversality condition

$$
\begin{array}{ll}
\dot{\lambda}_{1}=\xi \lambda_{1}\left(\ln \left(\frac{p_{*}(t)}{q_{*}(t)}\right)+1\right)+\lambda_{2}\left(\frac{2}{3} d \frac{q_{*}(t)}{p_{*}^{1 / 3}(t)}-b\right), & \lambda_{1}(T)=1, \\
\dot{\lambda}_{2}=-\xi \lambda_{1} \frac{p_{*}(t)}{q_{*}(t)}+\lambda_{2}\left(\mu+d p_{*}^{2 / 3}(t)+G u\right), & \lambda_{2}(T)=0,
\end{array}
$$

such that along $\left(\lambda(t), p_{*}(t), q_{*}(t)\right)$ the optimal control $u_{*}$ minimizes the Hamiltonian 


$$
H=\kappa u-\lambda_{1} \xi p \ln (p / q)+\lambda_{2}\left(b p-\left(\mu+d p^{2 / 3}\right) q-G u q\right)
$$

over the control set $[0, a]$ with the minimum value given by 0 . We call a pair $((p, q), u)$ consisting of an admissible control $u$ with corresponding trajectory $(p, q)$ for which there exists a multiplier $\lambda$ such that the conditions of the Maximum Principle are satisfied an extremal (pair), and the triple $((p, q), u, \lambda)$ is an extremal lift (to the cotangent bundle).

The conditions of the Maximum Principle show the close connection with the problem formulation (P1). The coefficient $\kappa$ replaces the constant multiplier $\lambda_{3}$ in (P1) and thus all essential properties that follow from these conditions remain preserved. A difference lies in the fact that $\kappa$ is constant for all trajectories whereas the value of the multiplier $\lambda_{3}$ generally varies from trajectory to trajectory for problem (P1). This needs to be considered if a synthesis, a solution for all initial conditions, is the aim. The two problems are closely connected, but not the same.

The minimization condition on the Hamiltonian $H$ is equivalent to minimizing the linear function $\left(\kappa-\lambda_{2}(t) G q_{*}(t)\right) v$ over $v \in[0, a]$. Thus, if we define the so-called switching function $\Phi$ as

$$
\Phi(t)=\kappa-\lambda_{2}(t) G q_{*}(t),
$$

then optimal controls satisfy

$$
u_{*}(t)= \begin{cases}0 & \text { if } \Phi(t)>0, \\ a & \text { if } \Phi(t)<0 .\end{cases}
$$

A priori the control is not determined by the minimum condition at times when $\Phi(t)=0$. However, if $\Phi(t) \equiv 0$ on an open interval, then also all derivatives of $\Phi(t)$ must vanish and this may determine the control. These are the so-called singular controls mentioned above. Generally, if singular controls can be excluded from the potential candidates for optimality, like it is the case, for example, for some models for cancer chemotherapy (e.g., $[21,22,35,33])$, then it is reasonable to expect that optimal controls will be bang-bang, that is, switch between the extreme points $u=0$ and $u=a$ of the control set. For this case optimization based sufficient conditions for local optimality exist (e.g., [28, 21]). However, for the problem considered here, singular controls indeed are optimal and directly applicable sufficient conditions for optimality are scarce and limited in scope (e.g., [15, 31]). Moreover, optimal controls first need to be synthesized from these candidates through an analysis of the switching function and this is the hard part of the analysis. This led to the concatenation sequence 0asa0 for problem (P1). Then, as in the calculus of variations, the construction of a field of extremals, a regular synthesis in the sense of Boltyanskii [6], verified the optimality of the solution. We also draw on these constructions for our claims of optimality for problem (P2). 
The analysis of the singular arc is analogous to the one for the 3-dimensional system (P1): If the control $u$ is singular on an open interval $I$, then $\Phi(t) \equiv 0$ and thus also the derivative of the switching function vanishes, i.e.,

$$
0 \equiv \dot{\Phi}(t)=-\dot{\lambda}_{2}(t) G q_{*}(t)-\lambda_{2}(t) G \dot{q}_{*}(t),
$$

and this simplifies to

$$
0=\left[\xi \lambda_{1}(t)-b \lambda_{2}(t)\right] G p_{*}(t) .
$$

Since $p_{*}(t)$ is positive it follows that $\xi \lambda_{1}(t)=b \lambda_{2}(t)$. Furthermore, the Hamiltonian vanishes identically and thus $\Phi(t) \equiv 0$ also implies that

$$
\lambda_{1}(t) \xi p(t) \ln \left(\frac{p(t)}{q(t)}\right)-\lambda_{2}(t)\left(b p(t)-\left(\mu+d p(t)^{2 / 3}\right) q(t)\right)=0
$$

and therefore

$$
\lambda_{2}(t)\left(b p(t)\left[\ln \left(\frac{p(t)}{q(t)}\right)-1\right]+\left(\mu+d p(t)^{2 / 3}\right) q(t)\right)=0 .
$$

From $\Phi(t) \equiv 0$ we get $\lambda_{2}(t) G q_{*}(t)=\kappa>0$, which implies that $\lambda_{2}(t)$ is positive along a singular arc. Thus the singular arc is given by the locus of all points $(p, q)$ that satisfy

$$
\mu+d p^{2 / 3} \equiv-b \frac{p}{q}\left(\ln \left(\frac{p}{q}\right)-1\right) .
$$

In the variables $p$ and $x=p / q$ this is exactly (10),

$$
p^{2}=-\left(\frac{b x(\ln x-1)+\mu}{d}\right)^{3} .
$$

The explicit formula (11) can be verified by differentiating $\dot{\Phi}(t) \equiv 0$ once more, and then solving for the control that explicitly appears in this derivative. However, the relation (21) will need to be used to simplify to the specified form.

This short computation is included to highlight for one case how the calculations and subsequent analysis are strongly related and in many instances identical with the computations done in [26] for problem (P1). As already mentioned, the reason is that the extra multiplier $\lambda_{3}$ in formulation (P1) associated with the constraint on $y$ is constant and in essence the two problems (P1) and (P2) become compatible if we set $\lambda_{3}=\kappa$. Hence the computations and subsequent analysis all carry over to problem formulation (P2). In particular, the singular curve and its admissible portion are given as in Proposition 2 and the concatenation structure of optimal controls is identical for both problems. This allows one to set up a direct minimization scheme to compute the optimal control for specified initial conditions. 
4. A numerical minimization scheme. The quantitative differences between problems (P1) and (P2) lie in how long optimal solutions stay on the singular arc. In formulation (P1) optimal controls typically follow the singular arc until all inhibitors have been exhausted. There are some exceptions if the singular control saturates at its upper value $a$ that are described in [26], but in this paper we only consider the most typical situation. Because of after-effects in the dynamics, optimal controls end with a segment with $u=0$ and the maximum tumor reduction is achieved at the time $T$ when the trajectory crosses the diagonal, $p(T)=q(T)$. For problem (P2) optimal trajectories leave the singular arc at some optimal time $\tau_{*}$ determined by the balance of tumor reduction and increase in the objective. While it is not possible to determine the optimal time $\tau_{*}$ analytically, it is not difficult to compute it numerically by introducing a 1-dimensional parameter $\tau$ that measures for how long the trajectory follows the optimal protocol for problem (P1). Depending on whether the point $(p(\tau), q(\tau))$ lies above or below the diagonal, either another segment for $u=0$ needs to be inserted until the diagonal $p=q$ is reached, or $\tau$ is the terminal time $T$. We then simply need to minimize the resulting parameterized objective.

We now formalize this procedure. For simplicity of presentation we restrict to one specific concatenation structure. The modifications for the other cases will be clear. We consider initial conditions $\left(p_{0}, q_{0}\right)$ for which the optimal control $u_{*}$ for formulation (P1) has the most typical form as0. That is, the control starts with an initial segment when inhibitors are given at maximum dose $a$ until the corresponding trajectory reaches the singular arc at time $\sigma_{1}$. Then the control becomes singular and the trajectory follows the singular arc until all inhibitors become exhausted at time $\sigma_{2}$ and the optimal control ends with a segment where no inhibitors are given until the diagonal $p=q$ is reached at time $\bar{T}$,

$$
u_{*}(t)= \begin{cases}a & \text { for } 0 \leq t \leq \sigma_{1}, \\ u_{\sin } & \text { for } \sigma_{1}<t \leq \sigma_{2}, \\ 0 & \text { for } \sigma_{2}<t \leq \bar{T} .\end{cases}
$$

Given such an initial condition $\left(p_{0}, q_{0}\right)$, let

$$
\bar{\gamma}=(\bar{p}, \bar{q}):[0, \bar{T}] \rightarrow \mathbb{R}_{+}^{2}
$$

denote the corresponding (P1)-optimal trajectory. The (P2)-value of this (P1)-optimal strategy is given by

$$
J_{2}\left(u_{*}\right)=\bar{p}(\bar{T})+\kappa A .
$$

Assuming that the tumor reduction achieved is smaller than the penalty on the inhibitors, $p_{0}-\bar{p}(\bar{T})<\kappa A$, it follows that $u_{*}$ is no longer optimal for problem (P2), simply because the trivial strategy of doing nothing already gives a better value for the objective. (We can always guarantee that $p_{0}-$ 
$\bar{p}(\bar{T})<\kappa A$ by choosing $A$ large enough, but the concatenation sequence may then change to asa0 if the singular control saturates.)

We now construct a 1-parameter family $\mathcal{F}$ of input-trajectory pairs $\left(p_{\tau}(\cdot), q_{\tau}(\cdot)\right)$ as follows: Initially follow the (P1)-optimal trajectory $\bar{\gamma}$ for time $\tau, 0 \leq \tau \leq \sigma_{2}$. If $p(\tau) \leq q(\tau)$, then no additional tumor reduction is possible along $u=0$ since $p$ would increase. Hence in this case we simply set $T(\tau)=\tau$. If $p(\tau)>q(\tau)$, then the tumor volume will still decrease along $u=0$ and as before we then switch the control to $u=0$ at time $\tau$ and follow the corresponding trajectory until the diagonal $p=q$ is reached at time $T=T(\tau)$,

$$
u_{\tau}(t)= \begin{cases}u_{*}(t) & \text { for } 0 \leq t \leq \tau \\ 0 & \text { for } \tau<t \leq T(\tau)\end{cases}
$$

The value of the corresponding objective is then given by

$$
I(\tau)=p_{\tau}(T(\tau))+\kappa \int_{0}^{\tau} u_{*}(t) d t .
$$

Since the qualitative structure of (P2)-optimal controls is the same as for problem $(\mathrm{P} 1)$, it follows that the (P2)-optimal trajectory is a member of this family. In fact, by construction all members are extremals. If $p_{0} \leq q_{0}$, then for $\tau=0$ this family formally contains the strategy of doing nothing and we simply get the initial tumor volume as value, $I(0)=p_{0}$. For $\tau=\sigma_{2}$ we obtain the (P1)-optimal trajectory, $I\left(\sigma_{2}\right)=\bar{p}(\bar{T})+\kappa A$. It is easily seen that the function $I:\left[0, \sigma_{2}\right] \rightarrow \mathbb{R}, \tau \mapsto I(\tau)$, is continuous and thus has a minimum over the compact interval $\left[0, \sigma_{2}\right]$. If the minimum occurs at $\tau_{*}<\sigma_{2}$, then the corresponding parameter defines the (P2)-optimal solution. If $\tau_{*}=\sigma_{2}$, then the original problem (P1) needs to be considered with a higher total limit $A$ on the inhibitors to find the (P2)-optimal solution. In fact, to begin with, one may simply compute the solution $\bar{\gamma}$ for problem (P1) so that $\sigma_{2}$ is the saturation time for the singular control. (For larger times $\sigma_{2}$ then the structure of optimal controls changes to asa0 and the construction of the family $\mathcal{F}$ would need to be modified.)

We illustrate the procedure with numerical results for the initial condition $p_{0}=q_{0}=15,000 \mathrm{~mm}^{3}$. Recall that for this initial condition we have $\sigma_{1}=0.04$ days, $\sigma_{2}=8.91$ days and $\bar{T}=9.09$ days so that the (P1)-optimal control $u_{*}$ is given by

$$
u_{*}(t)= \begin{cases}a & \text { for } 0 \leq t \leq 0.04 \\ u_{\sin } & \text { for } 0.04<t \leq 8.91 \\ 0 & \text { for } 8.91<t \leq 9.09\end{cases}
$$

Fig. 4 shows the graphs of the objective $I$ for various values of the weight $\kappa$ in the penalty term. Since for this initial condition the (P1)-optimal control 

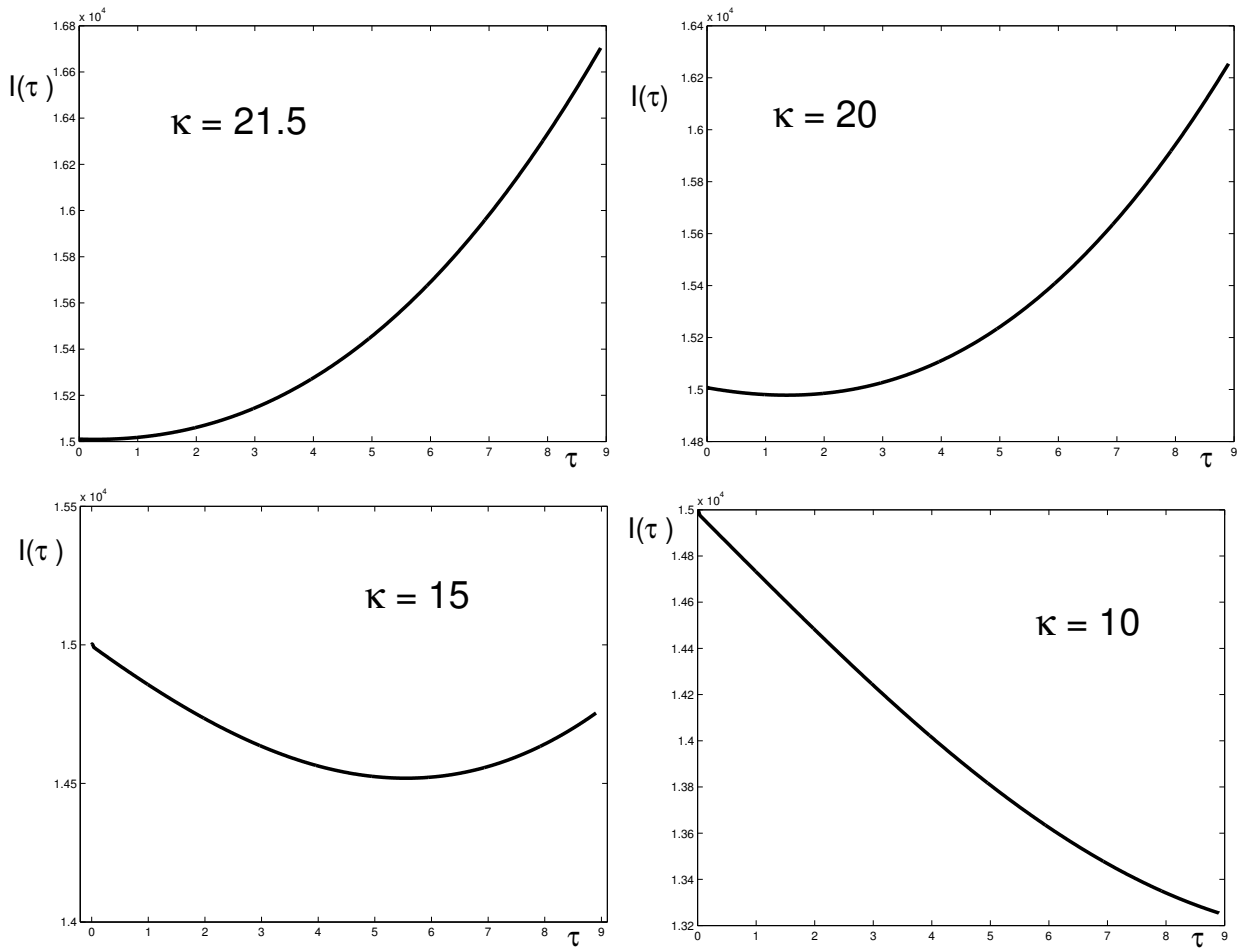

Fig. 4. Graphs of the function $I$ for $\kappa=21.5$ (a, top left), $\kappa=20$ (b, top right), $\kappa=15$ (c, bottom left), $\kappa=10$ (d, bottom right)

starts with $u=a$, the penalty term is dominant for large values of $\kappa$ and in these cases the function $I$ will be increasing, as shown for the case $\kappa=$ 21.5 in Fig. 4(a). This simply means that for this problem formulation and such a high weight, it would be "optimal" in the sense of minimizing the objective to do nothing, i.e., the best parameter value is given by the left end point $\tau=0$. This will be correct for all $\kappa>\kappa_{1}^{*}$ where the coefficient $\kappa_{1}^{*}$ is defined as the smallest value for which the optimal solution is still attained at the parameter $\tau_{1}^{*}=0$. As $\kappa$ decreases below $\kappa_{1}^{*}$, the structure of the (P2)-optimal control becomes $a 0$ and does not yet include a piece on the singular arc. Since the dynamics for $u=a$ is very fast - the (P1)-optimal control is at full dose only for time 0.04 days - for this initial condition this structure is only optimal over a tiny interval $\left(\kappa_{2}^{*}, \kappa_{1}^{*}\right)$ in $\kappa,\left(\kappa_{2}^{*}, \kappa_{1}^{*}\right)$ contained in $(20.75,21)$. The coefficient $\kappa_{2}^{*}$ is determined by the condition that the associated optimal parameter $\tau_{2}^{*}$ is given by the time $\sigma_{1}$ when the (P1)optimal control becomes singular, $\tau_{2}^{*}=\sigma_{1}=0.04$ days. Thus in this case the (P2)-optimal trajectory follows the $u=a$ trajectory until it hits the singular $\operatorname{arc} \mathcal{S}$ and then immediately bounces off with control $u=0$. As $\kappa$ 
decreases below $\kappa_{2}^{*}$, the minimum is achieved for a parameter $\tau>\tau_{2}^{*}$ and thus the structure of the (P2)-optimal control is now as0 as for problem (P1), but the optimal control follows the singular arc only until time $\tau$. For example, the graph of $I$ for $\kappa=20$ is shown in Fig. 4(b) and the minimum of $I$ is attained for $\tau=1.37$; the (P2)-optimal control is given by

$$
u(t)= \begin{cases}a & \text { for } 0 \leq t \leq 0.04 \\ u_{\sin } & \text { for } 0.04<t \leq 1.37 \\ 0 & \text { for } 1.37<t \leq T(1.37)\end{cases}
$$

including a small portion of the singular arc. This control and its corresponding trajectory are shown in Fig. 5. As $\kappa$ decreases further the parameter value for which the minimum is attained moves to the right and the interval along which the (P2)-optimal control is singular becomes longer. For example, for $\kappa=15$ the control is now singular until time $\tau=5.56$ as shown in Fig. 6 .
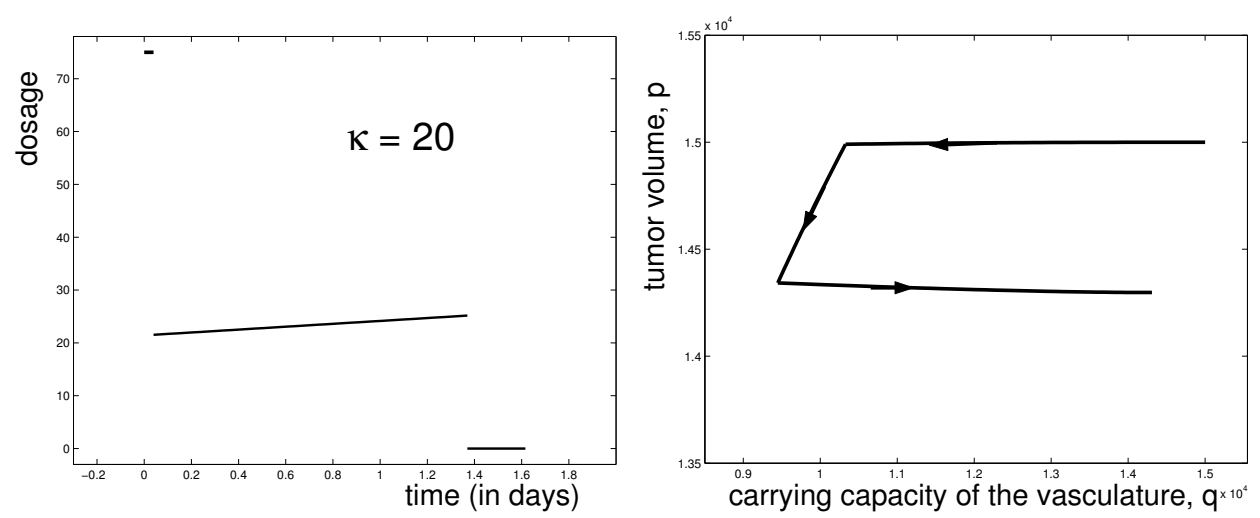

Fig. 5. (P2)-optimal control (left) and corresponding trajectory (right) for $\kappa=20$
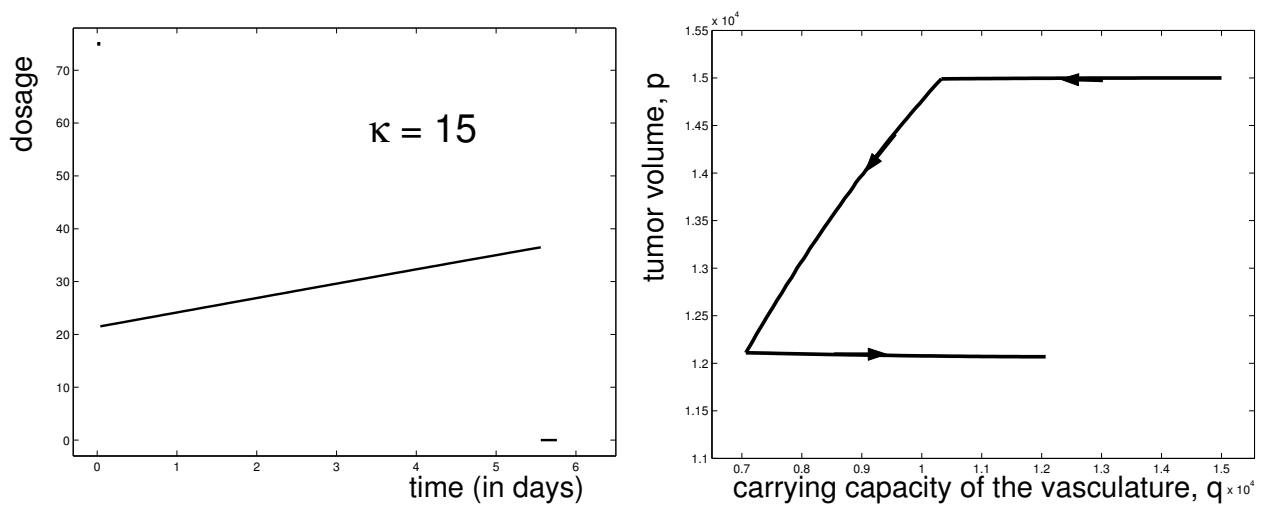

Fig. 6. (P2)-optimal control (left) and corresponding trajectory (right) for $\kappa=15$ 
Eventually, as $\kappa$ decreases further, for some value $\kappa_{3}^{*}$, the parameter value $\tau$ where the function $I$ attains its minimum becomes equal to the time $\tau_{3}^{*}=\sigma_{2}=8.91$ days when the singular control has used up exactly the amount $A$ given as constraint in problem (P1). In this case the (P1) and (P2)-optimal controls are identical. For $\kappa<\kappa_{3}^{*}$ the function $I$ is strictly decreasing on the interval $[0, \sigma]$ as shown in Fig. $4(\mathrm{~d})$ for $\kappa=10$. In this case the (P2)-optimal control uses more inhibitors than were allowed in model formulation (P1) and in order to solve problem (P2) we need to increase the limit $A$ in problem formulation (P1). For example, if we take $A=600$ here, then for $\kappa=10$ the upper limit $\sigma_{2}$ increases to $\sigma_{2}=14.68$ days and now the minimum is attained for $\tau=11.17$ inside the interval $\left[0, \sigma_{2}\right]$; see Fig. 7 . Also, for these values the singular control still does not saturate and thus the structure as0 is retained for the (P2)-optimal solution.

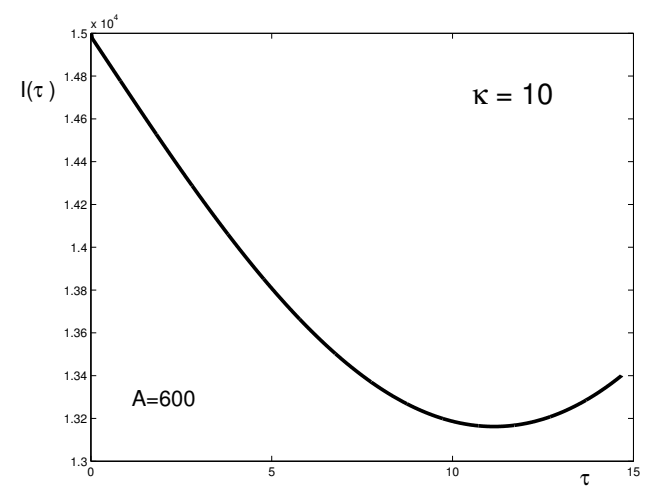

Fig. 7. Graph of the function $I$ for $\kappa=10$ with $A=600$

5. Conclusion. We described the solutions for two related optimal control problems for tumor anti-angiogenesis. Central to both solutions is an optimal singular arc $\mathcal{S}$, but the solutions differ in how long it is optimal to follow this curve. If the total amount of angiogenic inhibitors is imposed as a constraint (problem (P1)), then naturally all available drugs will be exhausted and therapy is ended as this limit is reached along $\mathcal{S}$. If the objective tries to achieve a balance between the amount of inhibitors given and their cost or side effects (problem (P2)), there exists a unique time when the benefits in tumor reduction from giving additional inhibitors are offset by negative side effects or cost as measured by the integral of the control. Based on the knowledge of the synthesis of (P1)-optimal controls and trajectories constructed in [26], the (P2)-optimal controls and trajectories can then easily be computed through a 1-dimensional numerical minimization procedure. 
Acknowledgements. This material is based upon research supported by the National Science Foundation under collaborative research grants DMS 0707404/0707410.

\section{References}

[1] Z. Agur, L. Arakelyan, P. Daugulis and Y. Ginosar, Hopf point analysis for angiogenesis models, Discrete Contin. Dynam. Systems Ser. B 4 (2004), 29-38.

[2] A. Anderson and M. Chaplain, Continuous and discrete mathematical models of tumor-induced angiogenesis, Bull. Math. Biol. 60 (1998), 857-899.

[3] L. Arakelyan, V. Vainstein and Z. Agur, A computer algorithm describing the process of vessel formation and maturation, and its use for predicting the effects of antiangiogenic and anti-maturation therapy on vascular tumour growth, Angiogenesis 5 (2002), 203-214.

[4] T. Boehm, J. Folkman, T. Browder and M. S. O'Reilly, Antiangiogenic therapy of experimental cancer does not induce acquired drug resistance, Nature 390 (1997), 404-407.

[5] B. Bonnard and M. Chyba, Singular Trajectories and their Role in Control Theory, Math. Appl. 40, Springer, 2003.

[6] V. G. Boltyanskii, Sufficient conditions for optimality and the justification of the dynamic programming principle, SIAM J. Control Optim. 4 (1966), 326-361.

[7] A. E. Bryson and Y. C. Ho, Applied Optimal Control, Hemisphere Publ., 1975.

[8] A. d'Onofrio, Rapidly acting antitumoral anti-angiogenic therapies, Phys. Rev. E 76 (2007), (3), art. 031920.

[9] A. d'Onofrio and A. Gandolfi, Tumour eradication by antiangiogenic therapy: analysis and extensions of the model by Hahnfeldt et al. (1999), Math. Biosci. 191 (2004), 159-184.

[10] S. Davis and G. D. Yancopoulos, The angiopoietins: Yin and Yang in angiogenesis, Curr. Top. Microbiol. Immunol. 237 (1999), 173-185.

[11] A. Ergun, K. Camphausen and L. M. Wein, Optimal scheduling of radiotherapy and angiogenic inhibitors, Bull. Math. Biol. 65 (2003), 407-424.

[12] J. Folkman, Antiangiogenesis: new concept for therapy of solid tumors, Ann. Surg. 175 (1972), 409-416.

[13] —, Angiogenesis inhibitors generated by tumors, Mol. Med. 1 (1995), 120-122.

[14] U. Foryś, Y. Keifetz and Y. Kogan, Critical-point analysis for three-variable cancer angiogenesis models, Math. Biosci. Engrg. 2 (2005), 511-525.

[15] H. Gardner-Moyer, Sufficient conditions for a strong minimum in singular control problems, SIAM J. Control 11 (1973), 620-636.

[16] P. Hahnfeldt, J. Folkman and L. Hlatky, Minimizing long-term burden: the logic for metronomic chemotherapeutic dosing and its angiogenic basis, J. Theor. Biol. 220 (2003), 545-554.

[17] P. Hahnfeldt, D. Panigrahy, J. Folkman and L. Hlatky, Tumor development under angiogenic signaling: a dynamical theory of tumor growth, treatment response, and postvascular dormancy, Cancer Res. 59 (1999), 4770-4775.

[18] R. S. Kerbel, A cancer therapy resistant to resistance, Nature 390 (1997), 335-336.

[19] -, Tumor angiogenesis: past, present and near future, Carcinogenesis 21 (2000), $505-515$. 
[20] M. Klagsburn and S. Soker, VEGF/VPF: the angiogenesis factor found?, Curr. Biol. 3 (1993), 699-702.

[21] U. Ledzewicz and H. Schättler, Optimal bang-bang controls for a 2-compartment model in cancer chemotherapy, J. Optim. Theory Appl. 114 (2002), 609-637.

[22] —, - Analysis of a cell-cycle specific model for cancer chemotherapy, J. Biol. Systems 10 (2002), 183-206.

[23] - - - A synthesis of optimal controls for a model of tumor growth under angiogenic inhibitors, in: Proc. 44th IEEE Conference on Decision and Control (Sevilla, 2005), 934-939.

[24] - - - Optimal control for a system modelling tumor anti-angiogenesis, ICGSTACSE J. 6 (2006), 33-39.

[25] - - - Application of optimal control to a system describing tumor anti-angiogenesis, in: Proc. 17th Int. Symposium on Mathematical Theory of Networks and Systems (MTNS) (Kyoto, 2006), 478-484.

[26] - - - Anti-angiogenic therapy in cancer treatment as an optimal control problem, SIAM J. Control Optim. 46 (2007), 1052-1079.

[27] - - - Optimal and suboptimal protocols for a class of mathematical models of tumor anti-angiogenesis, J. Theoret. Biol. 252 (2008), 295-312.

[28] H. Maurer, C. Büskens, J.-H. Kim and Y. Kaja, Optimization techniques for the verification of second-order sufficient conditions for bang-bang controls, Optimal Control Appl. Methods 26 (2005), 129-156.

[29] L. S. Pontryagin, V. G. Boltyanskii, R. V. Gamkrelidze and E. F. Mishchenko, The Mathematical Theory of Optimal Processes, MacMillan, New York, 1964.

[30] R. K. Sachs, L. R. Hlatky and P. Hahnfeldt, Simple ODE models of tumor growth and anti-angiogenic or radiation treatment, Math. Comput. Modelling 33 (2001), $1297 \mathrm{ff}$.

[31] G. Stefani, On sufficient optimality conditions for singular extremals, in: Proc. 42nd IEEE Conference on Decision and Control (CDC) (Maui, HI, 2003), 2746-2749.

[32] A. Świerniak, Direct and indirect control of cancer populations, Bull. Polish Acad. Sci. Tech. Sci. 56 (2008), 367-378.

[33] A. Świerniak and Z. Duda, Some control problems related to optimal chemotherapysingular solutions, Appl. Math. Comput. Sci. 2 (1992), 293-303.

[34] A. Świerniak, G. Gala, A. Gandolfi and A. d'Onofrio, Optimization of angiogenic therapy as optimal control problem, in: Proc 4th IASTED Conference on Biomechanics, M. Doblare (ed.), Acta Press, 2006, 56-60.

[35] A. Świerniak, U. Ledzewicz and H. Schättler, Optimal control for a class of compartmental models in cancer chemotherapy, Int. J. Appl. Math. Comput. Sci. 13 (2003), 357-368.

Department of Mathematics

and Statistics

Southern Illinois University at Edwardsville

Edwardsville, IL 62026-1653, U.S.A.

E-mail: uledzew@siue.edu
Department of Electrical and Systems Engineering

Washington University St. Louis, MO 63130-4899, U.S.A. E-mail: hms@wustl.edu 